\title{
Artérite temporale ou maladie de Horton : Guide clinique du professionnel des soins oculovisuels
}

\author{
William D. Kress, \\ optométriste, F.A.A.O. \\ Cincinnati Veterans Affairs \\ Medical Center
}

John M Neal, optométriste Central Arkansas Veterans Healthcare System

\section{RÉSUMÉ}

Objet Le présent article a pour but de passer en revue la problématique de l'artérite temporale, en mettant l'accent sur les manifestations ophtalmiques, et de fournir un guide de référence rapide pour l'identification clinique, le diagnostic et la prise en charge appropriée.

Résumé L'artérite temporale est l'une des rares urgences médicales qui peut amener un patient à se présenter initialement chez un professionnel des soins oculovisuels. La compréhension de l'évolution de la maladie et de sa prise en charge aidera le professionnel des soins oculovisuels à la détecter et à la prendre en charge tôt dans le processus, pouvant ainsi prévenir la cécité et des manifestations systémiques potentiellement mortelles.

\section{MOTS CLÉS}

Artérite temporale, neuropathie optique ischémique, pseudopolyarthrite rhizomélique, artère temporale, glucocorticoïde

\section{INTRODUCTION}

L'artérite temporale, aussi appelée la maladie de Horton, est classée comme une vascularite systémique qui affecte les vaisseaux moyens et les vaisseaux plus gros. ${ }^{1,2}$ L'artérite temporale est la plus commune des vascularites systémiques qui touchent principalement les personnes de plus de 50 ans. L'évolution clinique de l'artérite temporale, lorsqu'elle affecte le flux sanguin vers l'œil, se présente habituellement sous forme de perte de vision soudaine, unilatérale et indolore avec ou sans autre observation oculaire. L'artérite temporale touche les femmes trois fois plus que les hommes, avec une incidence estimée à 27 cas pour 100000 chez les personnes âgées de plus de 50 ans et une incidence maximale chez les personnes âgées de 70 à 80 ans. $^{3}$ Les personnes d'ascendance nord-européenne, en particulier scandinave, courent un risque plus élevé. ${ }^{4}$ L'artérite temporale suspectée est une urgence médicale et doit être prise en charge comme telle jusqu'à preuve du contraire. Outre une perte de vision importante, l'artérite temporale peut présenter d'autres manifestations oculaires et/ou manifestations systémiques, telles que maux de tête, claudication de la mâchoire, paralysie du nerf crânien, neuropathie périphérique, nécrose du cuir chevelu, altération de l'état mental, insuffisance cardiaque congestive, infarctus du myocarde, aortite, rupture d'anévrisme aortique et manifestations thromboemboliques..$^{5-14}$ Le présent article a pour but de fournir au professionnel des soins oculovisuels une mise à jour sur l'évolution clinique, le diagnostic et les options de traitement de l'artérite temporale. 
Étiologie et physiopathologie : L'étiologie et la pathogenèse exactes de l'artérite temporale demeurent inconnues. Cependant, plusieurs études ont démontré que l'étiologie peut être de nature multifactorielle et faire intervenir des facteurs génétiques et environnementaux. ${ }^{15-17}$ L'histopathologie de l'artère temporale montre des polyartérites segmentaires et focales avec inflammation non nécrosante. De plus, des lymphocytes T CD4+, des macrophages et des cellules géantes s'infiltrent dans les parois artérielles, suggérant ainsi une réaction immunitaire à un antigène déclencheur. ${ }^{18}$ Le virus de l'herpès zoster a été proposé comme antigène stimulant, mais la documentation n'est pas concluante. ${ }^{19}$ Même si un mécanisme spécifique est toujours insaisissable, le vieillissement s'accompagnant de changements physiologiques entraîne une réduction de la réaction immunitaire, une inflammation et une oxydation accrues, ainsi qu'une production accrue d'auto-anticorps, ce qui crée un environnement propice à une réaction auto-immune aux antigènes. ${ }^{20,21}$ Le processus inflammatoire résultant mène à une congestion vasculaire et à une ischémie tissulaire.

Évolution clinique : Environ un patient sur cinq chez qui on a diagnostiqué une artérite temporale ne présentera que des signes ou des symptômes oculaires, sans autre symptôme systémique. ${ }^{1}$ On estime qu'un patient sur trois présentant une artérite temporale aura des symptômes visuels transitoires (amaurose fugace) ou permanents. ${ }^{22} \mathrm{Il}$ a été signalé qu'une amaurose fugace avait touché de 10 à $30 \%$ des patients atteints d'artérite temporale; le pronostic visuel associé était moins favorable. Les symptômes de troubles visuels transitoires peuvent avoir un caractère unilatéral ou bilatéral. ${ }^{1,23}$

La perte de vision permanente, à divers degrés, se produit chez de 5 à $20 \%$ des patients pour lesquels une artérite

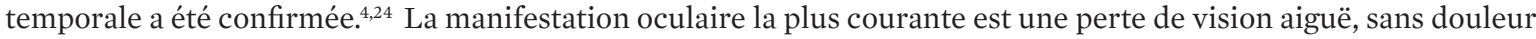
et unilatérale associée à la neuropathie optique ischémique. La neuropathie optique ischémique artéritique (AAION) touche près de $80 \%$ à $85 \%$ des personnes atteintes d'artérite temporale, et l'acuité peut varier de 20/200 à une incapacité totale de percevoir la lumière (figure $1^{1,24}$ ).

Figure 1 : Edème papillaire secondaire à une neuropathie optique ischémique artéritique

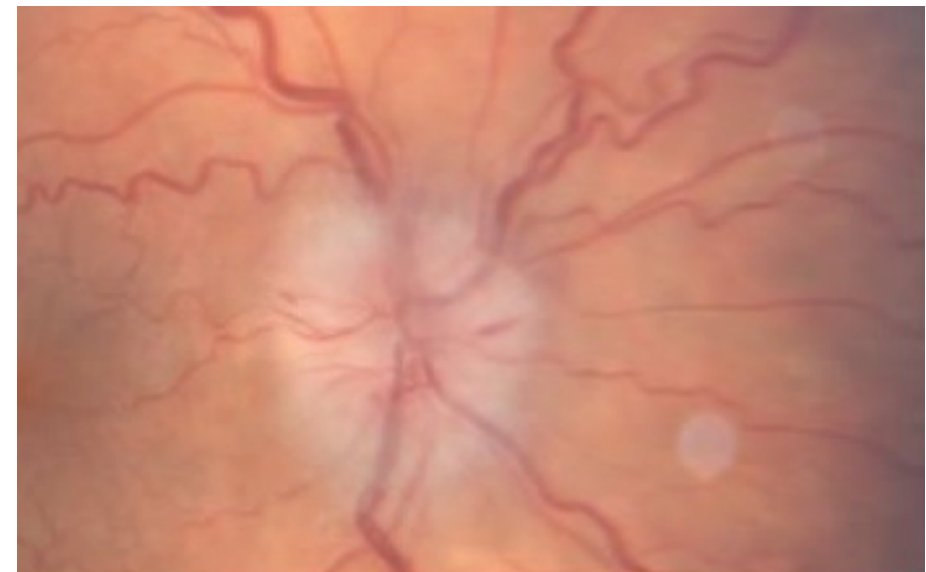

L'ischémie dans les artères ciliaires postérieures et les courtes branches alimentant la tête du nerf optique mène à l'apparition d'un signe clinique, soit un nerf œedémateux de couleur blanc crayeux avec ou sans hémorragie intrarétinienne environnante. L'atrophie optique se développe dans les 6 à 8 semaines suivant l'événement déclencheur et apparaît généralement comme une pâleur sectorielle sans autres résultats découlant de l'examen de la rétine. Un déficit pupillaire afférent sera présent dans les cas unilatéraux sans qu'il y ait eu d'événements rétiniens ou neurologiques antérieurs importants pour l'œil touché. Le champ visuel automatisé montre des signes d'une défectuosité altitudinale complète ou incomplète qui peut être ou non de nature absolue (figure 2). Bien que les défectuosités inférieures ou supérieures (relatives ou absolues) soient les défectuosités du champ visuel les plus couramment signalées, associées à la neuropathie optique ischémique, les défectuosités de motif peuvent revêtir de nombreuses apparences en fonction du type de champ (cinétique ou statique) ainsi que de la taille de l'essai (c.-à-d., de I-le à I-4e, V-4e). ${ }^{25}$

Bien que l'œdème discal puisse être plus diffus dans l'A-AION, l'évolution peut aussi être de nature sectorielle. La neuropathie optique ischémique postérieure artéritique (A-PION) est beaucoup moins répandue que l'A-AION et se présente de la même façon que l'A-AION, mais sans les résultats typiques fondoscopiques. À ce titre, l'A-PION est un diagnostic d'exclusion. 
Figure 2 : Déficit altitudinal inférieur absolu avec épargnes centrales, secondaire à une neuropathie optique ischémique

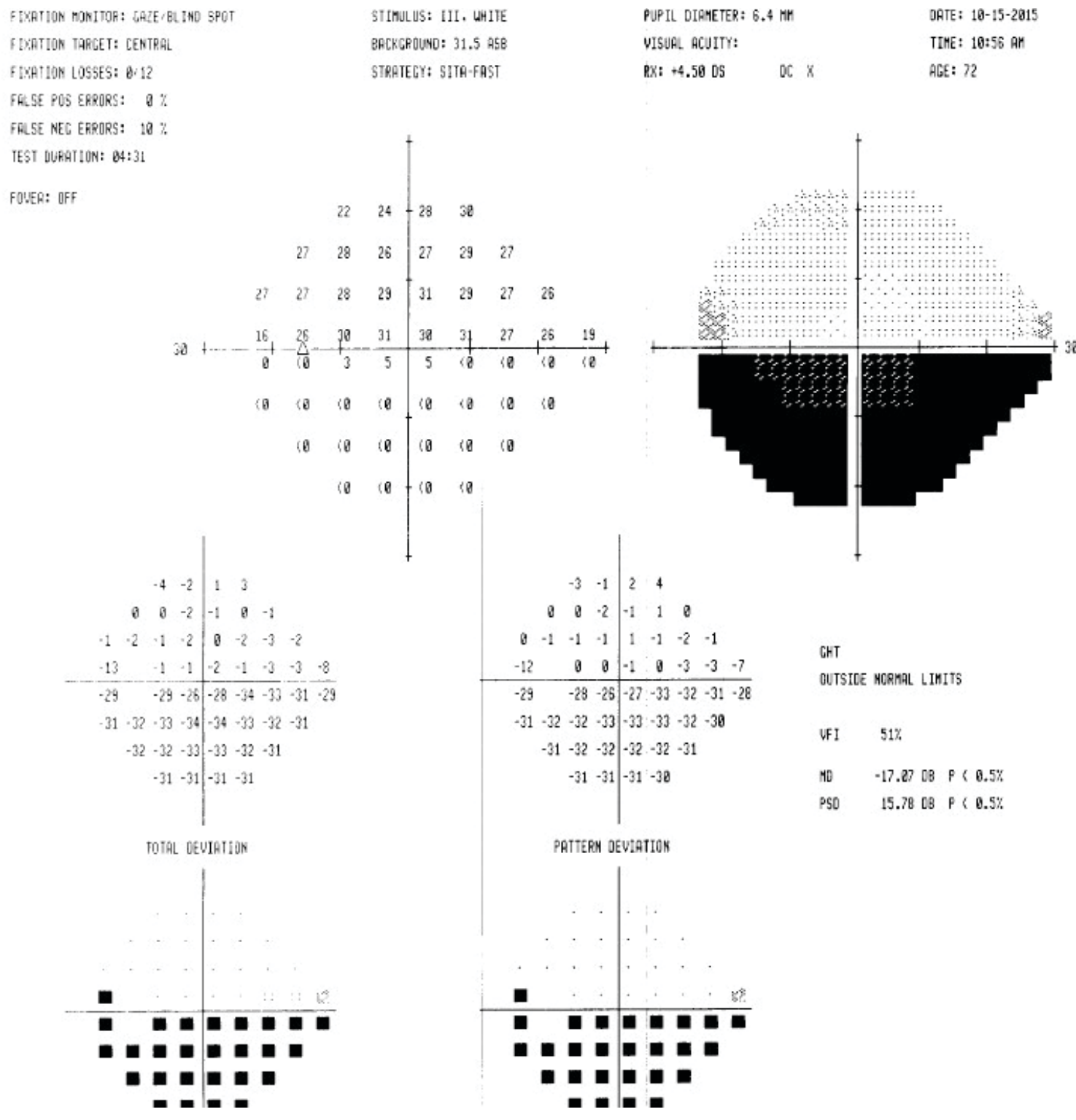

Parmi les autres possibilités d'évolution des observations oculaires associées à l'artérite temporale, mentionnons l'ischémie rétinienne (occlusion de l'artère rétinienne, occlusion de l'artère cilio-rétinienne, taches blanches ressemblant à de petits flocons de coton), l'ischémie choroïdienne (petites hémorragies rétiniennes et/ou changements de la pigmentaire rétinienne), l'ischémie du segment antérieur (hypotonie, œedème cornéen, uvéites, rubéose de l'iris), l'ophtalmoplégie (paralysie du nerf crânien, pseudo-tumeur de l'orbite), sclérite et syndrome d'ischémie oculaire. ${ }^{26}$

Bien que l'artérite temporale soit généralement caractérisée comme une maladie « de la tête et du cou », les manifestations systémiques devraient être explorées dans le cadre des antécédents d'une complication oculaire soupçonnée liée à l'artérite temporale chez le patient qui se présente au professionnel des soins oculovisuels. Les maux de tête sont le premier symptôme dans près de 32 à $40 \%$ des cas d'artérite temporale. ${ }^{27-29}$ L'évolution caractéristique sera une céphalée aiguë nouvellement apparue ou un changement dans la tendance des maux de tête antérieurs. La céphalée peut souvent être localisée et se produire dans la région temporale, dans 25 à $50 \%$ des manifestations. ${ }^{30}$ La céphalée peut être accompagnée d'une claudication de la mâchoire (douleur mandibulaire causée par un mouvement de mastication répétitif), d'une douleur dans la langue ou d'une douleur à la poitrine et d'odynophagie 
(douleur causée par l'ingestion d'aliments ou de liquides) en raison de l'aortite. L'artérite temporale peut être accompagnée de fièvre, d'une perte de poids (40\% des cas) ${ }^{31}$ et d'un malaise général.

Le tableau 1 présente d'autres manifestations systémiques associées à l'artérite temporale.

Tableau 1 : Autres manifestations systémiques de l'artérite temporale $e^{32-45}$

\begin{tabular}{|l|l|}
\hline Système & Signes et symptômes \\
\hline nerveux central & $\begin{array}{l}\text { accident ischémique transitoire } \\
\text { accident vasculaire cérébral } \\
\text { démence } \\
\text { neuropathie crânienne (autre qu'oculaire) }\end{array}$ \\
\hline nerveux périphérique & $\begin{array}{l}\text { mononeuropathies et polyneuropathies } \\
\text { nerf médian } \\
\text { nerfs C5-C6 }\end{array}$ \\
\hline cardiovasculaire & $\begin{array}{l}\text { aortite, dissection aortique, anévrisme aortique, sténose aortique } \\
\text { dissection ou anévrisme de l'aorte thoracique ou abdominale } \\
\text { sténose de l'artère cervicale, brachiale, subclavienne, axillaire ou de l'artère } \\
\text { de l'extrémité inférieure }\end{array}$ \\
\hline respiratoire & toux \\
\hline gastro-intestinal et rénal & $\begin{array}{l}\text { élévation des enzymes hépatiques } \\
\text { occlusion du petit intestin } \\
\text { maladie rénale et de la vessie (rare) }\end{array}$ \\
\hline reproductif & atteinte vasculaire du sein et des voies génitales féminines \\
\hline tégumentaire & nécrose du cuir chevelu, autrement rare \\
\hline
\end{tabular}

En ce qui concerne les manifestations systémiques de l'artérite temporale, la discussion doit inclure la pseudopolyarthrite rhizomélique (PMR) en raison de l'association apparente des deux processus morbides. Près de 40 à $60 \%$ des patients chez qui on a diagnostiqué une artérite temporale ont une PMR, et près de 16 à $21 \%$ des patients qui ont une PMR ont une artérite temporale. ${ }^{13,46-48}$ Le diagnostic de PMR est fondé sur des caractéristiques cliniques, notamment une douleur aiguë bilatérale aux épaules et une raideur et/ou une entorse pelvienne pendant plus de deux semaines. De plus, des symptômes constitutionnels tels que la fièvre, la fatigue, la perte de poids, la dépression et les sueurs nocturnes sont fréquents. ${ }^{46}$

\section{ÉTABLISSEMENT DU DIAGNOSTIC}

Les critères de l'American Academy of Rheumatology établis en 1990 pour la classification de l'artérite temporale sont actuellement utilisés comme norme pour le diagnostic de la maladie (tableau 2). La présence de trois des cinq critères ou plus présente un niveau de sensibilité de 93,5\% et un niveau de spécificité de 91,2\%. ${ }^{49}$

La combinaison des résultats de la vitesse de sédimentation (VS) et de la protéine C-réactive (CRP) augmente la spécificité d'un diagnostic d'artérite temporale par rapport à ce que l'on découvre avec l'un ou l'autre test. Certaines études ont montré que la CRP (> 2,45 mg/dL) seule est très sensible à cette condition. ${ }^{50,51}$ Notons que dans près de 4 à $14 \%$ des cas d'artérite temporale prouvés par biopsie de l'artère temporale, la VS et la CRP pourraient être normales. ${ }^{52,53}$ Plusieurs études suggèrent que la thrombocytose (augmentation du nombre de plaquettes) pourrait être un bon indicateur, conjuguée à la VS et à la CRP, dans le diagnostic de l'artérite temporale. ${ }^{51,54,55}$

Les tests en laboratoire et les symptômes des patients contribuent à prédire la probabilité d'artérite temporale. Toutefois, la biopsie de l'artère temporale (BAT) demeure le test standard pour l'artérite temporale prouvée cliniquement. La sensibilité diagnostique de la BAT est de 70 à $90 \% .{ }^{22}$ Chez les patients dont les résultats de la BAT sont négatifs, une investigation plus poussée de l'artérite temporale peut être indiquée dans les cas où les soupçons sont élevés d'après les travaux de laboratoire ainsi que d'après les signes et les symptômes cliniques. Pour les personnes atteintes de maladies des grands vaisseaux, telles que l'artérite temporale qui affecte l'aorte, les branches immédiates de l'aorte, les vaisseaux plus grands des membres supérieurs et inférieurs, ou chez les personnes pour lesquelles la suspicion d'artérite temporale est élevée malgré une BAT négative, il est suggéré dans la documentation récente que les techniques d'imagerie peuvent être utiles pour confirmer le diagnostic. ${ }^{56-59}$ Parmi les autres techniques de diagnostic de l'artérite temporale, mentionnons l'échographie Doppler, la tomographie par ordinateur et l'angiographie par tomodensitométrie, l'imagerie par résonance magnétique et l'angiographie par résonance magnétique ainsi que la tomographie par émission de positrons à l'aide du 18F-fluorodésoxyglucose (FDG). 
Tableau 2 : Critères de diagnostic de l'artérite temporale ${ }^{49}$

\begin{tabular}{|l|l|}
\hline System & Signs/Symptoms \\
\hline 1. Âge à l'apparition de la maladie, 50 ans et plus & L'apparition de symptômes ou de signes à partir de 50 ans ou plus \\
\hline 2. Nouveau mal de tête & Nouvel apparition ou nouveau type de douleur localisée dans la tête \\
\hline 3. Anomalies artérielles temporelles & $\begin{array}{l}\text { Sensibilité de l'artère temporelle à la palpation ou diminution des } \\
\text { pulsations, sans rapport avec l'artériosclérose des artères cervicales }\end{array}$ \\
\hline $\begin{array}{l}\text { 4. Vitesse élevée de sédimentation des } \\
\text { érythrocytes }\end{array}$ & $\begin{array}{l}\text { Vitesse de sédimentation des érythrocytes } 50 \text { mm/heure selon la méthode } \\
\text { Westergren }\end{array}$ \\
\hline 5. Biopsie anormale de l'artère & $\begin{array}{l}\text { Spécimen de biopsie de l'artère montrant une vascularite caractérisée par une } \\
\text { prédominance d'infiltration de cellules mononucléaires ou d'inflammation } \\
\text { granulomateuse, habituellement avec des cellules géantes multinucléées }\end{array}$ \\
\hline
\end{tabular}

PRISE EN CHARGE

Étant donné les résultats négatifs possibles de l'artérite temporale sur le plan visuel et systémique, un traitement médical est généralement amorcé à la suite d'un diagnostic de suspicion. Pour le professionnel des soins oculovisuels qui prend en charge un patient présentant des signes ou des symptômes d'artérite temporale, le plan d'action le plus probable sera d'aiguiller immédiatement le patient vers un spécialiste des soins oculovisuels tertiaires ou une salle d'urgence locale en indiquant la suspicion d'artérite temporale. Le cas échéant, une analyse sanguine superficielle est appropriée, et devrait surtout être axée sur une formule sanguine complète, la vitesse de sédimentation (VS) et la protéine C-réactive (CRP)

Un traitement aux glucocorticoïdes est le principal traitement d'une artérite temporale présumée ou prouvée par biopsie. Toutefois, le dosage et le régime optimaux pour le traitement initial et la prise en charge à long terme demeurent un sujet de débat. On cible davantage une prise en charge personnalisée. La pratique actuelle consiste à administrer de 20 à $60 \mathrm{mg} /$ jour de prednisolone afin de supprimer les symptômes liés à l'artérite temporale. Bien qu'il soit souhaitable de diminuer les glucocorticoïdes en raison des effets secondaires systémiques, le traitement peut se poursuivre pendant des mois, voire des années, selon la réponse individuelle au traitement. ${ }^{56,60-64}$

Pour l'artérite temporale présentant des symptômes visuels, on recommande actuellement un traitement aux glucocorticoïdes par voie intraveineuse (méthylprednisolone $1 \mathrm{~g} /$ jour pendant 3 jours consécutifs) avec de la prednisolone par voie orale ( $15 \mathrm{mg} / \mathrm{kg} /$ jour) et une diminution progressive du traitement par glucocorticoïdes oraux en fonction des signes et des symptômes du patient. ${ }^{65}$ Chez les patients qui ont déjà une déficience visuelle, un traitement plus précoce (dans les 24 heures) offre les meilleures chances de récupération de la vue. ${ }^{61,62,66}$ Les symptômes visuels sans signes évidents d'artérite temporale peuvent être initialement traités au moyen d'un traitement oral uniquement Comme pour le traitement des maladies non oculaires des grands vaisseaux sanguins, il n'existe pas de lignes directrices claires sur la posologie ou la durée efficace du traitement, et la prise en charge est personnalisée en fonction des signes et des symptômes du patient.

Comme pour tout traitement aux glucocorticoïdes, en particulier les soins de longue durée, les effets secondaires systémiques et le risque de complications associées au traitement aux stéroïdes soulèvent la question à savoir quelles sont les autres options de prise en charge de l'artérite temporale. Il a été démontré que l'administration sous-cutanée de tocilizumab approuvé par la FDA offre une efficacité immédiate et à long terme dans le traitement de l'artérite temporale. Plusieurs études ont montré que ce traitement était efficace et pouvait générer une rémission des signes et symptômes initiaux liés à l'artérite temporale. ${ }^{67-69}$ L'étude GiACTA a démontré que le tocilizumab est efficace pour la prise en charge de l'artérite temporale nouvelle ou récurrente, combinée à une diminution des glucocorticoïdes sur 52 semaines, où la dose globale de glucocorticoïdes est réduite au besoin pour prévenir les rechutes. ${ }^{70}$ Le rôle du tocilizumab dans la prise en charge des modifications de la vision associées à l'artérite temporale n'est pas clair pour le moment. Le traitement complémentaire peut inclure l'aspirine, les statines et/ou les antagonistes des récepteurs de l'angiotensine II en raison de leurs propriétés anti-inflammatoires et anticoagulantes et de leur potentiel pour éviter les rechutes, et cela s'est révélé bénéfique dans les études d'observation et les modèles animaux. ${ }^{71-75} \mathrm{Il}$ faut procéder à des essais cliniques aléatoires à grande échelle pour confirmer leurs rôles dans la prise en charge de l'artérite temporale. 


\section{PRONOSTIC}

Le pronostic de la perte de vision liée à l'artérite temporale est habituellement réservé à la manifestation initiale. Bien que l'on ait documenté un rétablissement important des événements de neuropathie optique ischémique ${ }^{75}$ la prise en charge vise à prévenir l'atteinte de l'autre œil, environ $50 \%$ des personnes étant touchées à l'autre œil dans les jours ou les semaines suivant l'événement initial. ${ }^{13,77}$ La rechute de l'artérite temporale est une préoccupation pour le professionnel de la santé responsable. On estime que de 43 à $80 \%$ des patients subissent une rechute de l'artérite temporale dans les deux années suivant l'événement initial. ${ }^{78-82}$ Même si les événements ischémiques, comme la neuropathie optique ischémique, ne sont pas caractéristiques dans les événements de rechute, les maux de tête, les symptômes PMR et les symptômes constitutionnels (myalgie, malaise, fièvre, nausées) peuvent être des manifestations d'une rechute. ${ }^{4,25,82}$

\section{CONCLUSION}

L'artérite temporale peut d'abord se présenter comme une perte de vision aiguë. Les signes ou les symptômes associés à la perte de vision liée à l'artérite temporale doivent être traités comme une urgence médicale. Le professionnel des soins oculovisuels doit prendre des mesures immédiates et évaluer le risque réel d'artérite temporale en fonction de ses observations. Si le diagnostic est incertain compte tenu de l'historique du patient, des résultats de l'examen ou de l'analyse sanguine superficielle, les manifestations oculaires devraient être traitées comme une artérite temporale jusqu'à preuve du contraire.

\section{REFERENCES}

1. Hayre SS, Podhajsky PA, Zimerman B. Occult giant cell arteritis ocular manifestations. Am J Ophthalmol 1998;125(4):521-6.

2. Ninan J, Lester S, Hill C. Giant cell arteritis. Best Pract Res Clin Rheumatol 2016;30(1):169-88.

3. Aiello PD, Trautmann JC, McPhee TJ, Kunselman AR, Hunder GG. Visual prognosis in giant cell arteritis. Ophthalmology 1993;100(4):550-5.

4. Carmona FD, Vaglio A, Mackie SL, et al. A genome-wide association study identifies risk of alleles in plasminogen and P4HA2 associated with giant cell arteritis. Am J Hum Genet 2017;100(10):64-74. doi. org/10.1016/j.ajhg.2016.11.013.

5. Save-Soderbergh J, Malmvall BE, Andersson R, Bengtsson BA. Giant cell arteritis as a cause of death. Report of nine cases. JAMA 1986;255:493-6.

6. Hupp SL, Nelson GA, Zimmerman LE. Generalized giant-cell arteritis with coronary involvement and myocardial infarction. Arch Ophthalmol 1990;108:1385-7.

7. Morris CR, Scheib JS. Fatal myocardial infarction resulting from coronary arteritis in a patient with polymyalgia rheumatic and biopsy-proven temporal arteritis. A case report and review of literature. Arch Intern Med 1994;154:1158-60.

8. Evans JM, O, Fallon WM, Hunder GG. Increased incidence of aortic aneurysm and dissection in giant cell(temporal)arteritis. A population-based study. Ann Intern Med 1995;122:502-7.

9. Caselli RJ, Daube JR, Hunder GG, Whisnant JP. Peripheral neuropathic syndromes in giant cell(temporal)arteritis. Neurology 1988;38:685-9.

10. Unwin B, Williams CM, Gilliland W. Polymyalgia rheumatic and giant cell arteritis. Am Fam Physician 2006;74:1547-54

11. Pascuzzi RM, Roos KL, Davis TE, Jr. Mental status abnormalities in temporal arteritis: a treatable cause of dementia in the elderly. Arthritis Rheum 1989;32:1308-11.

12. Weyand CM, Goronzy JJ. Giant cell arteritis and polymyalgia rheumatic. Ann Int Med 2003;139:505-15.

13. Hellman DB, Hunder GG. Giant cell arteritis and polymyalgia rheumatica. In: Harris ED, Budd RC, Genovese MC, Firestein GS, Sargent JS, Sledge CB (eds). Kelley's Textbook of Rheumatology, 7th ed. Philadelphia, PA: Elsevier Saunders;2005:1343-56.

14. Ross M, Bursztyn L, Superstein R, Gans M. Multiple cranial nerve palsies in giant cell arteritis. J Neuroophthalmol 2017;37(4):398-400.

15. Chen M, Gelman R, Al-Zubidi N, et al. Conjugal giant cell arteritis. J Neuro-Ophthalmol 2013;33:158-61.

16. Watts RA, Scott DG. Epidemiology of vasculitides. Semin Resp Crit Care Med 2004;25:455-64.

17. Smith CA, Fidler WJ, Pinals RS. Giant cell arteritis. Report of a ten-year study in Shelby County, Tennessee. Arthritis Rheum 1983;26:1214-9.

18. Buckingham EM, Foley MA, Grose C, et al. Identification of herpes zoster-associated temporal arteritis among cases of giant cell arteritis. Am J Ophthalmol 2018;187:51-60. doi.ord/10/1016/j.ajo.2017.12.017.
19. Lee HY, Oh BH. Aging and artery stiffness. Circ J 2010;74(11):2257-62.

20. Wang M, Monticone RE, lakatta EG. Arterial aging: a journey into subclinical arterial disease. Curr Opin Nephrol Hypertens 2010;19(2):201-7.

21. Borchers AT, Gershwin ME. Giant cell arteritis: a review of classification, pathophysiology, geoepidemiology and treatment. Autoimmun Rev 2012;11(6-7):A544-54.

22. Salvarini C, Cantini F, Boiardi L, Hunder GG. Polymyalgia rheumatica and giant cell arteritis. N Engl J Med 2002;347(4):261-7.

23. Chen JJ, Leavitt JA, Fang C, Crowson CS, Matteson EL, Warrington KJ. Evaluating the incidence of arteritis ischemic optic neuropathy and other causes from giant cell arteritis. Ophthalmology 2016;123(9):1999-2003. doi:10.1016/j.ophtha.2016.05.008.

24. De Smit E, O'Sullivan E, Mackey DA, Hewitt AW. Giant cell arteritis: ophthalmic manifestations of a systemic disease. Graefes Arch Clin Exp Ophthalmol 2016;254:2291-306.

25. Hayreh SS, Zimmerman B. Visual field abnormalities in non-arteritic ischemic optic neuropathy. Arch Ophthalmol 2005;123:1554-62.

26. Smeeth L, Cook C, Hall AJ. Incidence of diagnosed polymyalgia rheumatica and temporal arteritis in the United Kingdom, 1990-2001. Ann Rheum Dis 2006;65(8):1093- 8.

27. Gonzalez-Gay MA, Amoli MM, Garcia-Porrua C, Ollier WE. Genetic markers of disease susceptibility and severity in giant cell arteritis and polymyalgia rheumatica. Semin Arthitis Rheum 2003;33(1):38 48.

28. Caselli RJ, Hunder GG, Whisnant JP. Neurologic disease in biopsyproven giant cell(temporal)arteritis. Neurology 1988;38(3):352-9. doi. org/10.1212/WNL.38.3.352.

29. Solomon S, Cappa KG. The headache of temporal arteritis. J Am Geriatr Soc 1987;35(2):163-5. doi.org/10.1111/j.1532-5415.1987. tb01348.x.

30. Healey LA, Wilske KR. Presentation of occult giant cell arteritis. Arthritis Rheum 1980;23(6):641-3. doi.org/10.1002/art.1780230605.

31. Smith JH, Swanson JW. Giant cell arteritis. Headache 2014;54(8):1273-89.

32. Weyand CM, Goronzy JJ. Medium- and large-vessel vasculitis. N Engl J Med 2003;349(2):160-9. doi:10.1056/NEJMra022694.

33. Tang V, Fantaneanu T, Chakraborty S, Patel V, Dowlatshahi D. Intracranial non-occlusive thrombus and multiple strokes in giant cell arteritis. Can J Neurol Sci 2012;39(1):116-7.

34. Schon F. Involvement of smell and taste in giant cell arteritis. J Neurol Neurosurg Psychiatry 1988;51(12):1594.

35. Roomet A, Allen JS. Temporal arteritis heralded by facial nerve palsy. JAMA 1974;228(7):870-1. doi.org/10.1001/ jama.1974.03230320040027.

36. Solans-Laque R, Bosch-Gil JA, Molina-Catenario CA, Ortega-Aznar A, Alvarez-Sabin J, Vilardell-Tarres M. Stroke and multi-infarct dementia as presenting symptoms of giant cell arteritis: report of 7 cases and review of literature. Medicine 2008;87(6):335-44 
37. Nuenninghoff DM, Hunder GG, Christianson TJ, McClelland RL, Matteson EL. Mortality of large-artery complication(aortic aneurysm, aortic dissection, and/or large-artery stenosis)in patients with giant cell arteritis: a population-based study over 50 years. Arthritis Rheum 2003;48(12):3532-7.

38. Kermani TA, Warrington KJ, Crowson CS, et al. Large-vessel involvement in giant cell arteritis: a population-based cohort study of the incidence-trends and prognosis. Ann Rheum Dis 2013;72(12):1989-94.

39. Olopade CO, Sekosan M, Schraufnagel DE. Giant cell arteritis manifesting as chronic cough and fever of unknown origin. Mayo Clin Proc 1997;72(11):1048-50.

40. Killer HE, Holtz DJ, Kaiser HJ, Laeng RH. Diplopia, ptosis and hepatitis as presenting sign and symptoms of giant cell arteritis. $\mathrm{Br} \mathrm{J}$ Ophthalmol 2000;84(11):1319-20.

41. Burke AP, Sobin LH, Virmani R. Localized vasculitis of the gastrointestinal tract. Am J Surg Pathol 1995;19(3):338-49.

42. Kadotani Y, Enoki Y, Itoi N, Kojima F, Kato G, Lee CJ. Giant cell arteritis of the breast: a case report with review of literatures. Breast Cancer 2010;17(3):225-32. doil0.1007/s12282-009-0120-1.

43. Pradhan D, Amin RM, Jones MW, Surti U, Parwani AV. Giant cell arteritis of the female genital tract with occult temporal arteritis and marginal zone lymphoma harboring novel $20 \mathrm{q}$ deletion: a case report and literature review. Int J Surg Pathol 2016;24(1):78-84.

44. Gibson LE. Granulomatous vasculitides and the skin. Dermatol Clin 1990;8(2):335-45.

45. Kermani TA, Warrington KJ. Polymyalgia rheumatica. Lancet 2013;381(9860):63-72.

46. Salvarani C, Gabriel SE, O'Fallon WM, Hunder GG. The incidence of giant cell arteritis in Olmsted County, Minnesota: apparent fluctuations in a cyclic pattern. Ann Intern Med 1995;123(3):192-4.

47. Franzen P, Sutinen S, von Knorring J. Giant cell arteritis and polymyalgia rheumatica in a region of Finland: an epidemiologic. Clinica and pathologic study, 1984-1988. J Rheumatol 1992;19(2):273-6.

48. Hunder GG, Bloch DA, Michel BA, et al. The American Academy of Rheumatology 1990 criteria for the classification of giant cell arteritis. Arthritis Rheum 1990;33(8):1122-8.

49. Kermani TA, Schmidt J, Crowson CS, Ytterberg SR, Hunder GG Matteson EL, et al. Utility of erythrocyte sedimentation rate and C-reactive protein for the diagnosis of giant cell arteritis. Semin $\mathrm{Ar}$ thritis Rheum 2012;41(6):866-71. doi:10.1016/j.semarthrit.2011.10.005.

50. Walvick MD, Walvick MP. Giant cell arteritis: laboratory predictors of a positive temporal artery biopsy. Ophthalmology 2011;118(6):1201-4.

51. Salvarani C, Hunder GG. Giant cell arteritis with low erythrocyte sedimentation rate: frequency of occurrence in a population-based study. Arthritis Rheum 2001:45:140-5.

52. Parikh M, Miller NR, Lee AG, et al. Prevalence of a normal C-reactive protein with an elevated erythrocyte sedimentation rate in biopsyproven giant cell arteritis. Ophthalmology 2006;113:1842-5.

53. El-Dairi MA, Chang L, Proia AD, Cummings TJ, Stinnett SS, Bhatti MT. Diagnostic algorithm for patients with suspected giant cell arteritis. J Neuroophthalmol 2015;35(3):246-53

54. Oh LJ, Wong E, Andrici J, McClucskey P, Smight JEH, Gill AJ. The full blood count in giant cell arteritis. Intern Med J 2018 Apr;48(4):408-13.

55. Nina JW, Lester S, Hill CL. Giant cell arteritis: beyond temporal artery biopsy and steroids. Intern Med J 2017;47:1228-40.

56. Descamps L, Olagne L, Merlin C, Cachin F, Soubrier M, Mathieu S. Utility of PET/CT in the diagnosis of inflammatory rheumatic disease: a systematic review and meta-analysis. Ann Rheum Dis 2018 Nov;77(11):e81.

57. Buttgereit F, Dejaco C, Matteson EL, Dasgupta B. Polymyalgia rheumatica and giant cell arteritis. A systematic review. JAMA 2016;315(22):2442-58

58. Jacquier P, Wuarin R, Chizzolini C, Thumann G, Steffen H, Chronopoulos A. Case report: Masquerading large-vessel giant cell arteritis. Optom Vis Sci 2017;94(12):1159-64.

59. Labarca C, Koster MJ, Crowson CS, et al. Predictors of relapse and treatment outcomes in biopsy-proven giant cell arteritis: a retrospective cohort study. Rheumatology 2016;55:347-56.

60. Bienvenu B, Ly KH, Lambert M, et al. Management of giant cell arteritis: recommendations of the French study group for large vessel vasculitis(GEFA). Rev Med Intern 2016;37:154-65.

61. Dasgupta B, Borg FA, Hassan N, et al. BSR and BHPR guidelines for the management of giant cell arteritis. Rheumatology 2010;49:1594-7.
62. Dejaco S, Singh YP, Perel P, et al. European League Against Rheumatism. 2015 recommendations for the management of polymyalgia rheumatica: a European League Against Rheumatism/American College of Rheumatology collaborative initiative. Arthritis Rheumatol 2015;67(10):2569-80.

63. Mukhtyar C, Guillevin L, Cid MC, et al. European Vasculitis Study Group. EULAR recommendations for the management of large vessel vasculitis. Ann Rheum Dis 2009;68(3):318-23.

64. Frasier JA, Weyand CM, Newman NJ, Biousse V. The treatment of giant cell arteritis. Rev Neurol Dis 2008;5(3):140-52.

65. Mazlumzadeh M, Hunder GG, Easely KA, et al. Treatment of giant cell arteritis using induction therapy with high dose glucocorticoids: a double-blind, placebo-controlled, randomized prospective clinical trial. Arthritis Rheum 2006;54:3310-8.

66. Seitz M, Reichenbach S, Bonel HM, Adler S, Wermelinger F, Villiger PM. Rapid induction of remission of large vessel vasculitis by IL-6 blockade. A case series. Swiss Med Wkly 2011;141:w13156

67. Regent A, Redecker S, Deroux A, et al. Tocilizumab in giant cell arteritis: a multicenter retrospective study of 34 patients. J Rheumatol 2016;43(8):1547-52.

68. Maini RN, Taylor PC, Szechinski J, et al. Double-blind randomization controlled clinical trial of the interleukin- 6 receptor antagonist, tocilizumab, in European patients with rheumatoid arthritis who had an incomplete response to methotrexate. Arthritis Rheum 2006;54(9):2817-29.

69. Stone JH, Tuckwell K, Dimonaco S, et al. Trial of tocilizumab for giant cell arteritis. N Eng J Med 2017;377(4):317-28.

70. Schmidt WA. Seifert A, Gromnica-Ihle E, Krause A, Natusch A. Ultrasound of proximal upper extremity arteries to increase the diagnostic yield in large-vessel giant cell arteritis. Rheumatology 2008;47:96-101.

71. Souza AW, Okamoto KY, Abrantes F, Schau B, Bacchiega AB, Shinjo SK. Giant cell arteritis: a multicenter observational study in Brazil. Clinics 2013;68:317-22.

72. Scmhidt J, Kermani TA, Muratore F, Crowson CS, Matteson EL, Warrington KJ. Statin use in giant cell arteritis: a retrospective study. J Rheumatol 2013;40:910-5.

73. Pugnet G, Sailer L, Bourrel R, Montastruc JL, Lapeyre-Mestre M. Is statin exposure associated with occurrence or better outcome in giant cell arteritis? Results from a French population-based study. J Rheumatol 2015;42:316-22.

74. Alba MA, Garcia-Martinez A, Prieto-Gonzalez S, et al. Treatment with angiotensin II receptor blockers is associated with prolonged relapse-free survival, lower relapse rate, and corticosteroid-sparing effect in patients with giant cell arteritis. Semin Arthritis Rheum 2014;43:772-7.

75. Thurtell MJ, Kardon RH. Recovery of vision from no light perception in giant cell arteritis. Arch Ophthalmol 2012;130(8):1080-2.

76. Birkhead NC, Wagener HP, Schick RM. Treatment of temporal arteritis with adrenal corticosteroids: results in 55 cases in which lesion was proven at biopsy. JAMA 1958;163:821-7.

77. Bongartz T, Matteson EL. Large-vessel involvement in giant cell arteritis. Curr Opin Rheumatol 2006;18:10-7.

78. Cid MC, Garcia-Martinez A, Lozano E, et al. Five clinical conundrums in the management of giant cell arteritis. Rheum Dis Clin North Am 2007;33:819-34, vii.

79. Hoffman, GS, Cid MC, Hellmann DB, et al. A multicenter, randomized, double-blind, placebo-controlled trial of adjuvant methotrexate treatment for giant cell arteritis. Arthritis Rheum 2002;46:1309-18.

80. Jover JA, Hernandez-Garcia C, Morado IC, et al. Combined treatment of giant cell arteritis with methotrexate and prednisone. A randomized, double-blind, placebo-controlled trial. Ann Intern Med 2001;134:106-14

81. Alba, MA, Garcia-Martinez A, Prieto-Gonzalez S, et al. Relapse in patients with giant cell arteritis: Prevalence, characteristics, and associated clinical findings in a longitudinally followed cohort of 106 patients. Medicine 2014;93(5):194-201.

82. Martinez-Lado L, Calvina-Diaz C, Pineiro A, et al. Relapses and recurrences in giant cell arteritis: a population-based study of patients with biopsy-proven disease from northwestern Spain. Medicine 2011;90:186-93. 